\title{
Cannabiskonsum in der Schweiz: Prävalenz und Determinanten für Risikokonsum
}

\author{
M. Neuenschwander ${ }^{1}$ \\ U. Frick ${ }^{1,2}$ \\ G. Gmel ${ }^{3}$ \\ J. Rehm ${ }^{1,4}$
}

\author{
Cannabis Use in Switzerland: Prevalence Rates and Factors \\ Associated with Problematic Consumption
}

\section{Zusammenfassung}

Fragestellung: In diesem Artikel wird untersucht, welche Prävalenzen für Jugendliche und junge Erwachsene beim Cannabiskonsum in der Schweiz aktuell gelten, welche Faktoren für risikoreichen Cannabiskonsum bedeutsam sind und mit welchen Outcome-Faktoren Risikokonsum assoziiert ist. Methode: Die Analysen beruhen auf einer repräsentativen Schweizer Stichprobe von 5025 Jugendlichen und jungen Erwachsenen im Alter von 13 bis 29 Jahren, die im Rahmen eines Cannabis-Monitorings zu Gesundheit und Konsumverhalten befragt wurden. Ergebnisse: Die Lebenszeitprävalenz beträgt insgesamt $46 \%$, dabei liegt die Rate bei den Männern signifikant höher als bei den Frauen. Das Durchschnittsalter bei Erstkonsum liegt bei den 13- bis 18-Jährigen bei 14,7 Jahren. Ein Anteil von 13,2\% der befragten Personen konsumiert regelmäßig Cannabis, davon gehört ein Drittel zur Subgruppe mit einem risikoreichen Konsum. Als Faktoren, die signifikant mit Risikokonsum assoziiert sind, erweisen sich neben dem Geschlecht das Einstiegsalter und regelmäßiger Tabakkonsum. Schlussfolgerung: Künftige Aufklärungs- und Präventionsprogramme sollten prioritär auf Jugendliche zwischen 12 und 16 Jahren abgestimmt werden. Es besteht ein Bedarf an einem validen Cannabis-Screening-Instrument für die Identifikation von Personen mit Risikokonsum. Die Methode der Kurzintervention bietet sich als kosteneffektive und niederschwellige Maßnahme für deren Behandlung an.

Schliuisselwörter

Cannabis · Adoleszenz · Risikokonsum • Prävention · Kurzintervention

\section{Abstract}

Aim: To present data on the prevalence of current cannabis use among adolescents and young adults and to identify risk factors that predict problematic cannabis consumption and with which outcome factors this consumption is associated. Methods: The analyses are based on a representative sample of 5,025 Swiss adolescents and young adults aged between 13 to 29 years. The data concerning health and consumer behavior were obtained from subjects in the context of a cannabis monitoring project. Results: Lifetime prevalence amounts to $46 \%$, whereby men consume significantly more frequently than women. For the 13 to 18 year olds the age of onset for cannabis use averages at 14.7 years. A portion of $13.2 \%$ of all subjects consumes cannabis regularly out of which one third shows problematic consumption patterns. The following factors show a significant association with problematic cannabis consumption: Sex, age of onset and regular tobacco consumption. Conclusions: There is growing evidence for the need for educational, prevention and interventional programs tailored specifically to the 12 to 16 year olds. A valid screening method should be available in order to detect cannabis consumers at risk. The approach of brief intervention is recommended as a cost-effective measure to treat those at risk.

\section{Key words}

Cannabis · adolescents · problematic consumption · prevention . brief intervention

Institutsangaben

${ }^{1}$ Institut für Sucht- und Gesundheitsforschung, Zürich, Schweiz

2 Fachhochschule Technikum Kärnten, Österreich

${ }^{3}$ Schweizerische Fachstelle für Alkohol- und andere Drogenprobleme SFA, Lausanne, Schweiz

${ }^{4}$ University of Toronto, Kanada

Korrespondenzadresse

Dr. phil. M. Neuenschwander · Institut für Sucht- und Gesundheitsforschung ISGF · Konradstrasse 32 .

8031 Zürich ·Schweiz·E-mail: martin.neuenschwander@isgf.unizh.ch

Bibliografie

Suchttherapie 2005; 6: 126-132 @ Georg Thieme Verlag KG Stuttgart · New York

DOI $10.1055 / \mathrm{s}-2005-858618$

ISSN 1439-9903 
Einleitung

Cannabispräparate wie Marihuana und Haschisch gehören in den westlichen Gesellschaften zu den meistkonsumierten illegalen Drogen [1]. Dabei haben die Prävalenzraten des Cannabiskonsums in den vergangenen 10 Jahren vorwiegend bei Jugendlichen stark zugenommen. Die europäische Schulstudie ESPAD zeigt, dass in der Schweiz bereits rund ein Drittel der 15- bis 16-Jährigen Erfahrung mit Cannabiskonsum hat [2, 3]. Obwohl Handel und Konsum von Cannabis illegal sind, genießt diese Substanz insbesondere bei der jungen Generation ähnliche Toleranz und Akzeptanz wie die legalen Substanzen Tabak und Alkohol [4]. Der Popularitätsgrad von Cannabis dürfte auch damit zu tun haben, dass Cannabis nach wie vor überwiegend als „weiche“ Droge eingestuft wird, über längere Zeit auch wissenschaftlich eher ein Randthema darstellte und den Status einer zum Vergleich zu anderen Drogen erstaunlich unhinterfragten Toleranz genießt [5]. Dass die Substanz Cannabis neben dem Gebrauch als Rauschmittel auch über ein klinisch positives Potenzial zu verfügen scheint, beispielsweise bei der Behandlung von Übelkeit und Erbrechen, bei der Stimulation von Appetit und beim Einsatz als Analgetikum und Antispasmodikum [6, 7], trägt ebenfalls zum positiven Image bei. Die für den medizinischen Einsatz erforderlichen langzeitpharmakokinetischen Daten und Informationen stehen allerdings noch aus.

In jüngster Zeit ist Cannabis erneut zu einem zentralen Thema der Presse, der Fachwelt und der Gesundheitspolitik geworden. Denn nicht zuletzt im Zusammenhang mit dem starken Zuwachs an Wissen über die neurobiologischen Wirkmechanismen [8-10] verstärkt sich die Diskussion über mögliche negative Langzeitfolgen namentlich bei jungen Cannabiskonsumierenden. Im Fokus der wissenschaftlichen Diskussion stehen Reifungsdefizite bei jüngeren Menschen durch vermehrten Cannabiskonsum, die Gefahr der Abhängigkeit, kognitive Langzeitfolgen und das Risiko für psychotische Erkrankungen [11 - 14].

Die vorliegende Untersuchung hat zum Ziel, einen Beitrag zur Faktenlage zu leisten, was die Prävalenz und relevante Faktoren anbelangt, die mit risikoreichem Cannabiskonsum von Jugendlichen und jungen Erwachsenen zusammenhängen.

\section{Methoden}

\section{Studiendesign und Stichprobe}

Von Mai bis August 2004 wurden insgesamt 5025 zufällig ausgewählte Jugendliche und junge Erwachsene im Alter zwischen 13 und 29 Jahren zu Konsumverhalten und Gesundheit befragt. Zur Grundgesamtheit gehörten bei der Erstbefragung alle Einwohnerinnen und Einwohner der Schweiz im Alter von 13-29 Jahren, die in einer der drei Hauptlandessprachen (deutsch, französisch, italienisch) Auskunft geben konnten. Die Panelstudie ist so angelegt, dass dieselben Personen in einer Zweiterhebung zum gleichen Themenkreis erneut befragt werden, um Verläufe bestimmen zu können. Bei den Personen unter 15 Jahren wurde die Zustimmung eines Elternteils bzw. einer erziehungsberechtigten Person eingeholt. Das Ziehen der Stichprobe erfolgte in einem zweistufigen Auswahlverfahren, dabei wurden in einem ersten Schritt die Haushalte zufällig ausgewählt und in einem zweiten Schritt nach dem Zufallsprinzip die Personen bestimmt (höchstens ein Proband pro Haushalt).

Allgemeines Ziel der Cannabisstudie ist es, Ausmaß, Wirkung und psychosoziale Folgen von Cannabiskonsum bei Jugendlichen und jungen Erwachsenen zu untersuchen und Informationen über deren Einstellung zum Thema Cannabis zu erhalten.

\section{Datenerhebung und Instrumente}

Die Befragung der Probanden wurde mittels CATI (Computer Aided Telephone Interviewing) von einem dafür spezialisierten Marktforschungsinstitut durchgeführt. Das Interview enthielt insgesamt 103 Items, die ein weites Spektrum abdeckten mit Fragen zu Familien- und Wohnverhältnissen, zu Aus- und Weiterbildung, zur körperlichen und psychischen Gesundheit sowie zum Konsumverhalten von legalen und illegalen Substanzen. Knapp die Hälfte aller Fragen (46 Items) widmete sich dem Thema Cannabis, wobei neben Häufigkeit und Konsummenge ebenfalls Einstellungen zum eigenen Konsum erhoben wurden. Die Fragen zum Cannabis Use Disorders Identification Test CUDIT von Adamson und Sellman [15] wurden in der Übersetzung der jeweiligen Landessprache gestellt. Dabei handelt es sich um ein in Anlehnung an den Alcohol Use Disorders Identification Test AUDIT [16] entwickeltes Screeninginstrument, das der Identifikation von Personen mit risikoreichem Konsum dient. Der CUDIT umfasst 10 Items, womit die Konsumhäufigkeit, Fragen nach der typischen Dauer der Rauschwirkung, dem morgendlichen Konsum und zu verschiedenen Aspekten der wahrgenommenen Probleme im Zusammenhang mit dem Cannabiskonsum (wie Konzentrationsprobleme, Angst vor Kontrollverlust, Schuldgefühle und soziale Sanktionen) abgedeckt werden. Erhoben wurde der CUDIT nur bei Personen mit einem aktuellen Cannabisgebrauch (sog. User-Group).

\section{Operationalisierung der Studienvariablen}

Die im Folgenden in Klammern gemachten Prozentangaben beziehen sich auf die User-Group mit insgesamt 663 Personen (=100\%).

\section{Alkoholkonsum}

Der Alkoholkonsum wurde mit einer dreistufigen Variablen erfasst, dabei bildete Abstinenz die Baseline (10,5\%), mit der die erste Konsumstufe (80,9\%) mit maximal 3 Standarddrinks ${ }^{1}$ für Männer bzw. 2 Standarddrinks für Frauen täglich und die zweite Konsumstufe (4,5\%) mit mehr als 3 bzw. 2 Standarddrinks verglichen wurden. Die gewählten Grenzen basieren auf verschiedenen Risikokategorien, wie sie im angelsächsischen Raum häufig angewandt werden [17].

\section{Rauchverhalten}

Das Rauchverhalten wurde ebenfalls mit einer dreistufigen Variablen operationalisiert, wobei die Kategorien 1 Zigarette pro Tag (44,0\%), 2 bis 10 Zigaretten pro Tag (31,9\%) und mehr als 10 Zigaretten pro Tag $(24,1 \%)$ unterschieden wurden.

\footnotetext{
1 In Anlehnung an internationale Normen enthält ein Standarddrink 10-12 Gramm reinen Alkohol und entspricht $3 \mathrm{dl}$ Bier/Alkopops, $1 \mathrm{dl}$ Wein/Sekt oder $3 \mathrm{cl}$ Schnaps. Die konsumierte Alkoholmenge wurde
} anhand dieser Volumenangaben erfragt. 


\section{Urbanitätsgrad}

Die Differenzierung des Urbanitätsgrades erfolgte mit einer dichotomen Variablen, wobei zwischen Herkunftsorten der befragten Personen mit einer Bevölkerungszahl bis 10000 (= rural, 19,0\%) und über 10000 (= urban, $81 \%$ ) unterschieden wurde.

\section{Sprachregion}

Die unterschiedlichen Sprachregionen wurde dichotom erfasst, dabei unterschieden wir die deutschsprachige Region (67,7\%) von den lateinischsprachigen Regionen (französisch, italienisch und rätoromanisch) (32,3\%).

\section{Fehlzeiten}

Abwesenheiten während der letzten vier Wochen in der Schule oder am Arbeitsplatz wurden dreistufig gemessen mit den Kategorien 0 Tage (69,5\%), 1-2 Tage (14,6\%) bzw. 3 und mehr Tage $(15,9 \%)$.

\section{Problemhäufigkeit}

Die Probandinnen und Probanden wurden zu körperlichen und psychosozialen Problemen befragt, die sie explizit mit dem Cannabiskonsum in Zusammenhang bringen. Beispiele dafür sind: „Fühlte mich körperlich schlecht“, „Probleme mit Freunden/ Freundinnen“ oder „Probleme am Arbeitsplatz/in der Schule“. Da die Häufigkeiten bei den einzelnen Kategorien klein sind, bildeten wir einen Summenscore, der die psychosoziale Problemdichte widerspiegelt. Aufgrund der stark rechtsschiefen Verteilung wurde dieser Score umkodiert in eine Variable mit den Stufen „keine Probleme“ (77,8\%), „ein Problem“ (17,2\%) bzw. „zwei und mehr Probleme“ (5,0\%).

\section{Leistungsniveau}

Zur Einschätzung des eigenen schulischen bzw. beruflichen Leistungsniveaus diente eine 6-stufige Skala. Aus Gründen der schiefen Verteilung wurde diese Variable dichotomisiert und auf die Ausprägungen tiefes $(17,9 \%)$ vs. hohes $(82,1 \%)$ selbst berichtetes Leistungsniveau beschränkt.

\section{Cannabiskonsum}

$\mathrm{Zu}$ Vergleichszwecken werden die Lebenszeitprävalenzen für den Cannabiskonsum für die gesamte Stichprobe, d.h. für die 5025 (=100\%) befragten Personen, dargestellt.

Der aktuelle Konsum bezieht sich auf die 6-Monatsprävalenz mit vier Stufen: max. 1-mal pro Monat (36,1\%); 2- bis 4-mal pro Monat (30,0\%); 2 - bis 3-mal pro Woche (13,9\%) und 4-mal pro Woche oder häufiger $(20,0 \%)$.

Bei allen 10 CUDIT-Items beträgt der Range - also die Differenz zwischen maximalem und minimalem Wert - 4 Scorepunkte. Beim Aufaddieren aller Einzelwerte resultiert somit ein maximaler CUDIT-Score von 40 Punkten. In Anlehnung an die psychometrische Analyse von Adamson und Sellman [15] liegt der Cut-off für Risikokonsum bei 8 Scorepunkten. Damit zählen 213 Personen ( $=32,1 \%$ der User-Group) zur Gruppe mit einem risikoreichen Konsum. Die Risikogruppe zeichnet sich aus durch regelmäßigen, häufigen Konsum (die Hälfte konsumiert fast täglich Cannabis), wobei der Rauschzustand relativ lange dauert. Ein Drittel der Risikogruppe gibt an, bei einem typischen Konsum mindestens 3 bis 4 Stunden berauscht zu sein. Die Zu- gehörigkeit zur Risikogruppe figuriert in den multivariaten Analysen als abhängige Variable.

\section{Statistische Analyse}

Bivariate Zusammenhänge wurden mittels $\chi^{2}$-Tests auf Unabhängigkeit geprüft. Multivariate logistische Regressionen dienten zur Selektion von prädiktiven Einflussfaktoren, die bedeutsam mit risikoreichem Cannabiskonsum in Zusammenhang stehen (CUDIT-Score von 8 oder mehr). Die Stärke der Assoziationen zwischen den Outcome-Variablen (Fehlzeiten, Problemdichte, Leistungsniveau) und risikoreichem Cannabiskonsum wurde mitttels Phi-Koeffizient bzw. Kendall's Tau bestimmt.

Um auf die gesamte Schweiz bezogene Aussagen machen zu können, wurden die Daten für die Berechnungen jeweils nach Alter, Geschlecht, Region, Haushalts- und Agglomerationsgröße gewichtet. Die Berechnungen erfolgten mit SPSS 12.0 [18].

\section{Resultate}

\section{Prävalenzen und Einstiegsalter}

Die Lebenszeitprävalenz von Cannabiskonsum beträgt für die männlichen Befragten 52,2\% und für die weiblichen 39,6\% (Tab.1), wobei 7,3\% der Männer und 8,6\% der Frauen angeben, lediglich einmal Cannabis konsumiert zu haben; die Geschlechter unterscheiden sich beim Lebenszeitkonsum signifikant.

Wie erwähnt, konstituiert sich die von uns vertieft untersuchte User-Group aus Probanden mit aktuell regelmäßigem Cannabiskonsum ( $n=663$ ). Die Konsumhäufigkeiten auf der 4-stufigen Skala fallen bei den Geschlechtern insgesamt nicht unterschiedlich aus, hingegen gibt es einen signifikanten Unterschied zwischen den zwei gebildeten Altersgruppen (13- bis 18-Jährige vs. 19- bis 29-Jährige). Dabei sind die Unterschiede beim seltenen und beim häufigen Konsum deutlich: Bei den 13- bis 18-Jährigen konsumieren $48,0 \%$ seltener als einmal monatlich, bei den 19- bis 29-Jährigen sind es $31,7 \%$. Hingegen konsumieren $22,6 \%$ dieser Altersgruppe häufig (viermal pro Woche oder häufiger) gegenüber $12,8 \%$ bei der jüngeren Altersgruppe. Wie erwartet, ist Cannabiskonsum stark assoziiert mit Tabak- und Alkoholkonsum. Insgesamt berichten nur rund $10 \%$ der Cannabis-User von Alkoholabstinenz gegenüber rund $80 \%$, die angeben, täglich bis zu 3 Standarddrinks (Männer) bzw. 2 Standarddrinks (Frauen) zu konsumieren. Die cannabiskonsumierenden Männer trinken signifikant mehr als die Frauen, ebenfalls liegen die Werte in der älteren Altersgruppe höher als bei der jüngeren. Beim Tabakkonsum sind die Werte ausgeglichener. Gar nicht oder höchstens eine Zigarette pro Tag zu rauchen betrifft $43,9 \%$ der cannabiskonsumierenden Frauen und Männer, rund ein Drittel raucht höchstens 10 und ein Viertel mehr als 10 Zigaretten pro Tag. Geschlechtsdifferenzen sind nicht nachweisbar. Die Prävalenzwerte liegen bei den 19- bis 29-Jährigen signifikant höher als bei den 13- bis 18-Jährigen, da die Raucherkarriere der jüngeren Probanden erst am Anfang steht.

Das durchschnittliche Einstiegsalter beträgt bei den 13 - bis 18-Jährigen 14,7 Jahre, bei den 19- bis 29-Jährigen 16,4 Jahre (Tab.1). Die durchgeführte Kaplan-Meier-Analyse zeigt überdies, dass betreffend Einstiegsalter ein Kohorteneffekt feststellbar ist, 
Tab. 1 Prävalenzwerte von Cannabis-, Alkohol- und Tabakkonsum bei regelmäßigem Konsum insgesamt sowie getrennt nach Geschlecht und Altersgruppen (Angaben in \%)

\begin{tabular}{|c|c|c|c|c|c|c|c|c|c|}
\hline & gesamt & männlich & weiblich & $\chi^{2}$ & $p$ & $\begin{array}{l}\text { Alter } \\
13-18\end{array}$ & $\begin{array}{l}\text { Alter } \\
19-29\end{array}$ & $\chi^{2}$ & Sig. \\
\hline \multicolumn{10}{|l|}{$\begin{array}{l}\text { gesamte Stichprobe } \\
(n=5,025)\end{array}$} \\
\hline \multicolumn{10}{|l|}{ Cannabiskonsum } \\
\hline Lifetime & 46,0 & 52,2 & 39,6 & 78,9 & 0,000 & 27,3 & 55,4 & 360,3 & 0,000 \\
\hline $1 \times$ & 7,9 & 7,3 & 8,6 & & & 6,8 & 8,5 & & \\
\hline 2 - bis $5 \times$ & 13,0 & 13,2 & 12,7 & & & 9,2 & 14,9 & & \\
\hline $6+$ & 25,1 & 31,7 & 18,3 & 386,3 & 0,000 & 11,3 & 32,0 & 386,3 & 0,000 \\
\hline \multicolumn{10}{|l|}{ User-Group $(n=663)$} \\
\hline \multicolumn{10}{|l|}{ Cannabis } \\
\hline \multicolumn{10}{|l|}{ 6-Monats-Prävalenz } \\
\hline$<1 /$ Monat & 36,1 & 33,4 & 42,0 & & & 48,0 & 31,7 & & \\
\hline 2-4/Monat & 30,0 & 31,9 & 25,9 & & & 25,1 & 32,0 & & \\
\hline $2-3 /$ Woche & 13,9 & 14,2 & 13,2 & & & 14,0 & 13,7 & & \\
\hline $4+/$ Woche & 20,0 & 20,6 & 18,9 & 4,92 & 0,177 & 12,8 & 22,6 & 17,84 & 0,000 \\
\hline \multicolumn{10}{|l|}{ Alkoholkonsum } \\
\hline \multicolumn{10}{|c|}{ 12-Monats-Prävalenz } \\
\hline abstinent & 10,9 & 8,6 & 15,8 & & & 16,1 & 8,9 & & \\
\hline$\leq 3$ (2) Drinks/Tag & 84,4 & 87,7 & 77,3 & & & 82,8 & 85,0 & & \\
\hline > 3 (2) Drinks/Tag & 4,7 & 3,7 & 6,9 & 11,33 & 0,003 & 1,1 & 6,1 & 12,59 & 0,000 \\
\hline \multicolumn{10}{|l|}{ Tabakkonsum } \\
\hline \multicolumn{10}{|l|}{ aktueller Konsum } \\
\hline$<1$ Zig./Tag & 43,9 & 43,9 & 43,9 & & & 56,1 & 39,4 & & \\
\hline 2-10 Zig./Tag & 32,0 & 30,2 & 35,8 & & & 28,9 & 33,0 & & \\
\hline > 10 Zig./Tag & 24,1 & 25,9 & 20,3 & 3,38 & 0,185 & 15,0 & 27,6 & 17,60 & 0,000 \\
\hline Einstiegsalter & $15,9 \pm 2,0$ & $16,0 \pm 1,9$ & $15,8 \pm 2,2$ & & & $14,7 \pm 1,3$ & $16,4 \pm 2,0$ & & \\
\hline
\end{tabular}

und zwar in dem Sinn, dass in der Gruppe der 13- bis 18-Jährigen der Einstieg signifikant früher erfolgt als in der Gruppe der 19bis 29-Jährigen (Log-Rank = 23,2, p= 0,000; Abb.1).

\section{Prädiktoren für risikoreichen Cannabiskonsum}

Um zu untersuchen, mit welchen Faktoren riskanter Cannabiskonsum (d.h. CUDIT-Summenwerte von 8 und mehr) wie stark assoziiert ist, wurde eine logistische Regression durchgeführt (Tab. 2). Als relevante Einflussgrößen resultieren das Geschlecht, das Einstiegsalter in den regelmäßigen Konsum und der Grad des Tabakkonsums. Männer tragen im Vergleich zu den Frauen ein erhöhtes Risiko, zur Gruppe von Probanden mit einem risikoreichen Cannabiskonsum zu gehören ( $O R=1,9$, 95\%-KI 1,3-2,9). Ein späterer Konsumbeginn wirkt protektiv ( $O R=0,8,95 \%-K I$ 0,8-0,9), d.h., erfolgt der Erstkonsum um ein Jahr später, sinkt das Chancenverhältnis, einen riskanten Konsum zu entwickeln, um 20\%. Auch Raucherinnen und Raucher sind gegenüber Nichtrauchern deutlich einem erhöhten Risiko ausgesetzt, zu den Problemkonsumenten zu gehören. Dabei steigt das Risiko linear mit der konsumierten Anzahl Zigaretten pro Tag. Für Raucher mit einem Tageskonsum von maximal 10 Zigaretten ist das Chancenverhältnis gegenüber der Referenzgruppe der Nichtraucher um das knapp 3fache erhöht $(\mathrm{OR}=2,8$, $95 \%-K I$ 1,8-4,3). Für (starke) Raucher mit einem Tageskonsum von mehr als 10 Zigaretten erhöht sich das Chancenverhältnis auf das 5fache (OR=5,1, 95\%-KI 3,2-8,1). Regelmäßiger Alkoholkonsum ist insgesamt mit risikoreichem Cannabiskonsum assoziiert, wobei die Irrtumswahrscheinlichkeit mit $\mathrm{p}=0,057$ knapp über der 5\%-Grenze liegt. Der Vorzeichenwechsel bei den Regressionskoeffizienten der beiden Konsumstufen hat mit dem kurvilinearen Risikoverlauf (J-Shape-Phänomen) zu tun, wonach die Risikokurve beim Alkoholkonsum nicht linear mit der konsumierten Menge steigt, sondern ein Minimum bei einem geringen Alkoholkonsum aufweist [19]. Die Kontraste zwischen den beiden Konsumstufen und Alkoholabstinenz als Referenz fallen nicht signifikant aus. Die Größe des Wohnortes und die Sprachregion, aus der die untersuchten Cannabis-User stammen, üben keinen bedeutsamen Einfluss auf die Zugehörigkeit zur Risikogruppe aus. Das dargestellte Modell erklärt 19\% der Varianz und 76,2\% der Fälle werden richtig klassiert.

\section{Outcome-Faktoren}

Die selbst berichteten psychosozialen Probleme sind signifikant mit risikoreichem Konsum assoziiert ( $\tau=0,35$, Tab. 3 ). Dies hat zum Teil mit der Konfundierung der Merkmalsoperationalisierung zu tun, denn Probleme im Zusammenhang mit Cannabiskonsum sind Bestandteil des CUDIT, daher ist eine positive Korrelation zur subjektiven Problemdichte zu erwarten. Keine Assoziationen lassen sich allerdings zwischen Risikokonsum und Fehlzeiten bzw. dem selbst eingeschätzten Leistungsniveau in der Schule und am Arbeitsplatz feststellen. Hingegen sind Fehlzeiten und die Anzahl an psychosozialen Problemen negativ mit dem Leistungsniveau assoziiert. 


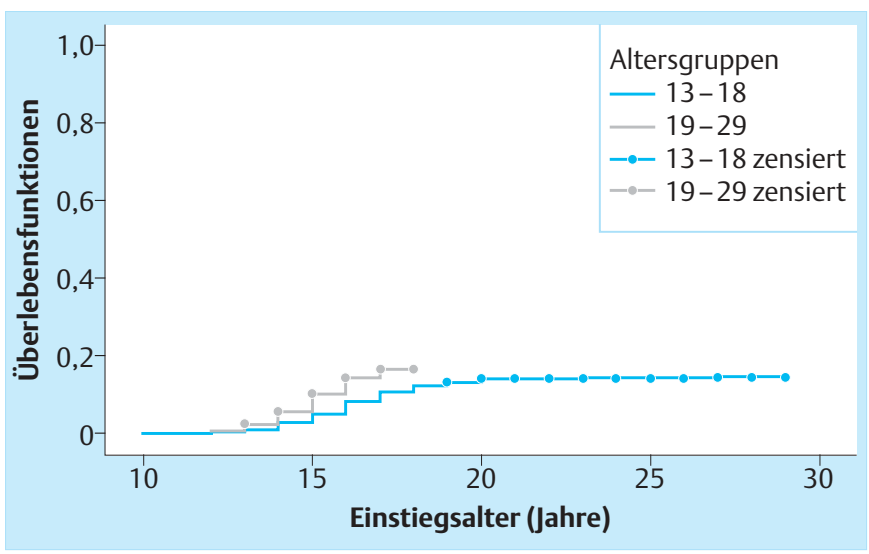

Abb. 1 Kumulativ-komplementäre Überlebensfunktionen für das Einstiegsalter nach Altersgruppen (Log-Rank-Wert =23,2, p=0,000).

Tab. 2 Multiple logistische Regression zur Vorhersage von risikoreichem Cannabiskonsum durch ausgewählte Einflussfaktoren

\begin{tabular}{|c|c|c|c|c|c|}
\hline Prädiktor & Kodierung & Beta & Sig. & OR & $95 \%-K I$ \\
\hline Geschlecht & männlich/weiblich & 0,652 & 0,002 & 1,9 & $1,3-2,9$ \\
\hline $\begin{array}{l}\text { Beginn des } \\
\text { Konsums }\end{array}$ & Jahre & $-0,172$ & 0,001 & 0,8 & $0,8-0,9$ \\
\hline Alkoholkonsum & & & 0,057 & & \\
\hline Referenz & Abstinenz & & & 1,0 & \\
\hline $\begin{array}{l}\text { Alkoholkonsum } \\
\text { Niveau I }\end{array}$ & $\leq 3$ (2) Drinks/Tag & $-0,189$ & 0,523 & 0,8 & $0,5-1,5$ \\
\hline $\begin{array}{l}\text { Alkoholkonsum } \\
\text { Niveau II }\end{array}$ & $>3$ (2) Drinks/Tag & 0,799 & 0,103 & 2,2 & $0,9-5,8$ \\
\hline Tabakkonsum & & & 0,000 & & \\
\hline Referenz & <1 Zig./Tag & & & 1,0 & \\
\hline $\begin{array}{l}\text { Tabakkonsum } \\
\text { Niveau I }\end{array}$ & $<2-10$ Zig./Tag & 1,016 & 0,000 & 2,8 & $1,8-4,3$ \\
\hline $\begin{array}{l}\text { Tabakkonsum } \\
\text { Niveau II }\end{array}$ & > 10 Zig./Tag & 1,627 & 0,000 & 5,1 & $3,2-8,1$ \\
\hline Urbanitätsgrad & $>10000$ & 0,040 & 0,877 & 1,0 & $0,6-1,7$ \\
\hline Sprachregion & deutsche/lat. Schweiz & 0,318 & 0,118 & 1,4 & $0,9-2,0$ \\
\hline
\end{tabular}

Anmerkungen: Varianzaufklärung nach Nagelkerke $R^{2}=0,190$; die Trefferquote beträgt $76,2 \%$

Tab. 3 Bivariate Korrelationen (berechnet mittels Kendall's Tau bzw. Phi-Koeffizient) zwischen risikoreichem Cannabiskonsum und ausgewählten Outcome-Faktoren

\begin{tabular}{llll}
\hline Outcome-Faktoren & Fehlzeiten & Problemdichte & Leistungsniveau \\
\hline Risikokonsum & Tau $=-0,02$ & Tau $=0,35$ & $\mathrm{Phi}=0,06$ \\
& $\mathrm{p}=0,562$ & $\mathrm{p}=0,000$ & $\mathrm{p}=0,180$ \\
\hline Fehlzeiten & & Tau $=0,02$ & Tau $=-0,23$ \\
& & $\mathrm{p}=0,520$ & $\mathrm{p}=0,000$ \\
Problemdichte & & & Tau $=-0,11$ \\
& & & $\mathrm{P}=0,019$ \\
\hline
\end{tabular}

Anmerkungen: Die Outcome-Faktoren wurden wie folgt kodiert: Fehlzeiten: (1) 0 Tage, (2) 1 bis 2 Tage, (3) 3 Tage und länger;

Problemdichte: (1) kein Problem, (2) ein Problem, (3) 2 und mehr Probleme; Leistungsniveau: (1) tief, (2) hoch.

( $p=$ Irrtumswahrscheinlichkeit)
Diskussion

Der Cannabiskonsum ist in der Schweiz im internationalen Vergleich sehr hoch. Das betrifft sowohl die Lebenszeitprävalenz von $46 \%$ bei den 13- bis 29-Jährigen als auch den Anteil von Personen mit einem sechs- oder mehrmaligen Konsum, der in dieser Untersuchung bei $25 \%$ liegt. Ähnliche Prävalenzraten werden in der jüngsten europäischen ESPAD-Studie genannt [2] und scheinen den Trend des verstärkten Cannabiskonsums fortzusetzen, wie er aus der Schülerstudie HBSC [20] und der Schweizer Multizenterstudie SMASH bereits bekannt ist [21]. Ähnliche Werte werden aus weiteren aktuellen Cannabisstudien berichtet [22-24].

Alkohol- und Tabakkonsum stehen bei dieser Untersuchung nicht im Mittelpunkt, werden aber im Sinn einer umfassenderen Beurteilung des Konsumverhaltens mit berücksichtigt. In dieser Untersuchung beträgt die Alkoholabstinenz in der Altersgruppe der 13- bis 18-Jährigen 16,1\% und sie liegt damit etwas höher als die Referenzwerte aus den erwähnten Studien ESPAD, HBSC und SMASH $[2,20,21]$. Gut $60 \%$ der 19- bis 20-Jährigen und knapp $44 \%$ der 13- bis 18-Jährigen rauchen regelmäßig. Auch diese Werte bestätigen das aktuelle (hohe) Konsumniveau in der Schweiz. Bei Alkohol-, Tabak- und Cannabiskonsum handelt es sich um die von der jungen Generation am häufigsten konsumierten Substanzen. Es ist bekannt, dass sich die Problembereiche gerade beim Konsum dieser Substanzen stark überschneiden $[22,25,26]$.

Die durchgeführte logistische Regressionsanalyse zeigt, dass neben dem Geschlecht das Alter bei Beginn des regelmäßigen Cannabiskonsums und das Rauchverhalten als bedeutsame Faktoren in Erscheinung treten. Weder die Sprachregion noch ein ländlicher bzw. städtischer Wohnort sind signifikant mit risikoreichem Cannabiskonsum assoziiert.

Bedeutsame Geschlechtsunterschiede beim Konsumverhalten können bei allen Altersstufen festgestellt werden, dabei handelt es sich um einen bereits bekannten Befund [2]. Die Unterschiede haben unter anderem mit einer geschlechtsspezifischen Risikowahrnehmung zu tun und können ebenfalls in anderen Verhaltensbereichen wie beim Fahrverhalten $[27,28]$ oder im Sport festgestellt werden [29].

Aus unserer Sicht zentral ist der Befund, dass ein früher Erstkonsum mit einer erhöhten Chance einhergeht, zur Risikogruppe zu gehören. Der Befund, dass das Einstiegsalter für Cannabiskonsum gegenüber Anfang der 90er-Jahre gesunken ist [30, 31], kann anhand unserer Daten bestätigt werden. In der Gruppe der 13- bis 18-Jährigen liegt das Einstiegsalter bereits unter 15 Jahren und damit signifikant niedriger als in der Gruppe der 19- bis 29-Jährigen. Dieser Kohorteneffekt ist insofern von Bedeutung, als dass sich in der Literatur die Befunde mehren, wonach früher und regelmäßiger Cannabiskonsum insbesondere in der auch hirnphysiologisch sensiblen pubertären Phase zu möglicherweise irreversiblen emotionalen und kognitiven Langzeitfolgen mit negativen Auswirkungen auf das Kurzzeitgedächtnis, die Informationsverarbeitung und das Problemlösungsverhalten führt $[9,11,12,32,33]$. Ob allenfalls Toleranzgrenzen für risikofreien Cannabiskonsum festgelegt werden können, bleibt abzuwarten. 
Die Gruppe der regelmäßig Cannabis Konsumierenden wurde anhand der 6-Monatsprävalenz ermittelt. Die auf diese Weise gefundene User-Group umfasst 13,2\% der Gesamtstichprobe. Diese Quote ist vergleichbar mit anderen Studien; so berichten Coffey et al. [22] in ihrer australischen Cannabisstudie einen User-Anteil von 14,4\% und gemäß der schweizerischen Gesundheitsbefragung 2002 weisen in der Schweiz 16,3\% der jungen Männer im Alter zwischen 15 und 24 Jahren und 7,7\% der jungen Frauen aktuellen Cannabiskonsum auf [34].

Im Hinblick auf primär- und sekundärpräventive Maßnahmen sind die Konsummuster von entscheidender Bedeutung. Bei der Definition von „Risikokonsum“ hielten wir uns aus pragmatischen Gründen an den Schwellenwert von 8 Scorepunkten beim Cannabis Use Disorders Identification Test (CUDIT) von Adamson und Sellman [15]. Diese Autoren legten den Cut-off in ihrer psychometrischen Untersuchung bei 8 Punkten fest, um in ihrem Sample Probanden mit Cannabisabhängigkeit zu identifizieren. Beim CUDIT handelt es sich um ein neues Screeninginstrument, das noch relativ wenig bekannt ist und den eigentlichen Bewährungstest erst noch bestehen muss. Der Bedarf nach einem validen Screeninginstrument ist deshalb groß, weil sich nur auf diese Weise eine bedarfsgerechte Primär- und Sekundärprävention etablieren lassen. Perkonigg et al. [24] fanden in ihrer prospektiven Langzeitstudie mit Jugendlichen, dass mit steigender Konsumhäufigkeit zu Beginn des Cannabiskonsums die Chance umso geringer ausfiel, mit dem Cannabiskonsum später wieder aufzuhören. Dieses Resultat steht im Widerspruch zur Annahme, dass es sich bei frühem Cannabiskonsum vorwiegend um einen vorübergehenden Probierkonsum handelt $[2,35]$ und legt den Schluss nahe, die Primärprävention gezielt auf Jugendliche zwischen 12 und 16 Jahren vorzuverlegen, um damit eine Früherfassung zu ermöglichen bzw. bei entsprechender Indikation sekundärpräventiv eine Kurzintervention (brief intervention) anbieten zu können. Bei Kurzinterventionen handelt es sich um zielgerichtete Interventionen von ein bis drei Sitzungen, in denen an die Klienten wesentliche Informationen abgegeben werden, die Motivation zur Verhaltensveränderung abgeklärt sowie psychische und soziale Ressourcen aktiviert werden [36, 37]. Seit über 20 Jahren werden bei Alkoholproblemen Kurzinterventionen durchgeführt und es besteht eine hinreichende Evidenz für deren Wirksamkeit [38]. Die Erfahrungsbasis im Drogenbereich ist noch nicht so groß, aber entsprechende empirische Studien weisen ebenfalls grundsätzlich eine erwünschte Wirksamkeit nach $[39,40]$.

Es dürfte sich lohnen, in künftigen Forschungsvorhaben die Früherkennung von problematischem Cannabiskonsum weiterzuentwickeln und zu validieren, damit insbesondere gefährdete Jugendliche rechtzeitig erfasst und von auf ihre Bedürfnisse zugeschnittenen Informations-, Beratungs- und Behandlungsangeboten profitieren können.

\section{Literatur}

${ }^{1}$ Watson S, Benson J, Joy J. Marijuana and medicine: assesseing the science base: a summary of the 1999 Institute of Medicine report. Archives of General Psychiatry 2000; 57: 547-552
${ }^{2}$ Gmel G, Rehm J, Kuntsche E et al. European School Survey Project on Alcohol and Other Drugs (ESPAD) in Switzerland: Wichtigste Ergebnisse 2003 und aktuelle Empfehlungen. Lausanne \& Zürich: Schweizerische Fachstelle für Alkohol- und andere Drogenprobleme (SFA), 2004

${ }^{3}$ Hibell B, Andersson B, Bjarnason T et al. The ESPAD Report 2003 - Alcohol and Other Drug Use Among Students in 35 European Countries. Stockholm: The Swedish Council for Information on Alcohol and Other Drugs, CAN Council of Europe, Co-operation Group to Combat Drug Abuse and Illicit Trafficking in Drugs (Pompidou Group), 2004

${ }^{4}$ Kuntsche E, Delgrande J, Schmid H. Wird Cannabis von 15-Jährigen in der Schweiz wie Tabak und Alkohol konsumiert? Ein faktorenanalytischer Ansatz. Wiener Zeitschrift für Suchtforschung 2002; 25 (4): $17-25$

${ }^{5}$ Täschner KL. Cannabisbezogene Störungen: Ein zentrales Thema der nächsten Jahre? Sucht 2004; 50 (5): 288 - 289

${ }^{6}$ Kalant H. Medical use of cannabis: history and current status (review). Pain Res Manag 2001; 6 (2): 80-91

${ }^{7}$ Rommelspacher H. Pharmakologische Verwendung von Cannabinoiden als Medikamente. Sucht 2004; 50 (5): 290 - 296

${ }^{8}$ Ameri A. The effects of cannabinoids on the brain. Progress in Neurobiology 1999; 58: $315-348$

${ }^{9}$ Solowij N. Long-term effects of cannabis on the central nervous system In: Kalant H, Corrigal W, Smart R (Hrsg). The Health Effects of Cannabis. Toronto: ARF Books, 1999: 195-265

${ }^{10}$ Wallmichrath I, Szabo B. Cannabinoide und neurale Signalübertragung. Sucht 2004; 50 (5): 297-308

${ }^{11}$ Schneider M, Koch M. Chronic pubertal, but not adult chronic cannabinoid treatment impairs sensorimotor gating, recognition memory and the performance in a progressive ratio task in adult rats. Neuropsychopharamacology 2003; 28: 1760-1769

12 Pope H, Gruber A, Hudson J et al. Early-onset cannabis use and cognitive deficits: what is the nature of the association? Drug and Alcohol Dependence 2003; 69: 303-310

${ }^{13}$ Arseneault L, Cannon M, Witton J et al. Causal association between cannabis and psychosis: examination of the evidence. Br J Psychiatry 2004; 184: $110-117$

${ }^{14}$ Schaub M, Rossler WRS. Cannabis and psychosis - a review. Schweiz Rundsch Med Prax 2004; 93 (23): 997-1002

${ }^{15}$ Adamson S, Sellman J. A prototype screening instrument for cannabis use disorder: the Cannabis Use Disorders Identification Test (CUDIT) in an alcohol-dependent clinical sample. Drug and Alcohol Review 2003; 22: 309-315

${ }^{16}$ Saunders JB, Aasland O, Babor R et al. Development of the Alcohol User Disorders Identification Test (AUDIT): WHO Collaborative Project on Early Detection of Persons with Harmful Alcohol Consumption. Addiction 1993; 88: $791-804$

17 White I, Altmanan D, Nanchahal K. Mortality in England and Wales attributable to any drinking, drinking above sensible limits and drinking above lowest-risk level. Addiction 2004; 99 (6): 749-756

18 SPSS. 12.0 Command Syntax Reference. Chicago: SPSS Inc, 2004

${ }^{19}$ Rehm J, Gutjahr E, Gmel G. Alcohol and all-cause mortality: A pooled analysis. Contemporary Drug Problems 2001; 28 (3): 337-363

${ }^{20}$ Schmid H, Graf M, Delgrande Jordan M et al. Trends im Konsum psychoaktiver Substanzen von Schülerinnen und Schülern in der Schweiz. Lausanne: Schweizerische Fachstelle für Alkohol- und andere Drogenprobleme, 2003

21 Narring F, Tschumper A, Inderwildi Bonivento L et al. Santé et styles de vie des adolescents âges de 16 à 20 ans en Suisse (2002). SMASH 2002: Swiss multicenter adolescent study on health 2002. Lausanne, Bern, Bellinzona: Institut universitaire de médicine sociale et préventive/Institut für Psychologie/Sezione sanitaria, 2003

22 Coffey C, Carlin J, Lynskey M et al. Adolescent precursors of cannabis dependence: findings from the Victorian Adolescent Health Cohort Study. Br J Psychiatry 2003; 182 (4): 330-336

${ }^{23}$ Coffey C, Carlin JB, Degenhardt L et al. Cannabis dependence in young adults: an Australian population study. Addiction 2002; 97 (2): $187-194$

24 Perkonigg A, Lieb R, Höfler M et al. Patterns of cannabis use, abuse and dependence over time: incidence, progression and stability in a sample of 1228 adolescents. Addiction 1999; 94 (11): 1663-1678

25 Taylor R, Fergusson D, Milne B et al. A longitudinal study of the effects of tobacco and cannabis exposure in young adults. Addiction 2002; 97: $1055-1061$ 
${ }^{26}$ Kalant H. Adverse effects of cannabis on health: an update of the literature since 1996. Progress in Neuro-Psychopharmacology \& Biological Psychiatry 2004; 28: 849-863

27 Begg D, Langley J. Identifying predictors of persistent non-alcohol or drug-related risky driving behaviours among a cohort of young adults. Accident Analysis and Prevention 2004; 36: 1067 - 1071

28 Glendon A, Dorn L, Davis D et al. Age and gender differences in perceived accident likelihood and driver competences. Risk Anal 1996; 16 (6): $755-762$

${ }^{29}$ Kontos A. Perceived risk, risk taking, estimation of ability and injury among adolescent sport partcipants. Journal of Pediatric Psychology 2004; 29 (6): $447-455$

${ }^{30}$ Müller S, Geml G. Veränderungen des Einstiegsalters in den Cannabiskonsum: Ergebnisse der zweiten Schweizer Gesundheitsbefragung 1997. Soz Präventivmed 2002; 47: 1 - 10

${ }^{31}$ Patton G, McMorris B, Toumbourou J et al. Puberty and the onset of substance use and abuse. Pediatrics 2004; 114 (3): 300-306

32 Bolla K, Brown K, Eldreth D et al. Dose-related neurocognitive effects of marijuana use. Neurology 2002; 59: 1337-1343

33 Schneider M. Langfristige Folgen des chronischen Cannabiskonsums. Sucht 2004; 50 (5): 309-319

${ }^{34}$ Bundesamt für Statistik. Schweizerische Gesundheitsbefragung 2002. Erste Ergebnisse unter www.bfs.admin.ch
${ }^{35}$ McCambridge J, Strang J. Age of first use and ongoing patterns of legal and illegal drug use in a sample of young Londoners. Subst Use Misuse 2005; 40 (3): $313-319$

${ }^{36}$ Rist F, Demmel R, Hapke U et al. Riskanter schädlicher und abhängiger Alkoholkonsum: Screening, Diagnostik, Kurzintervention. Sucht 2004; 2: $102-112$

37 Steinberg K, Roffman R, Carrol K et al. Tailoring cannabis dependence treatment for a diverse population. Addiction 2002; 97: 135- 142

${ }^{38}$ Moyer A, Finney J, Swearingen C et al. Brief interventions for alcohol problems: a meta-analytic review of controlled investigations in treatment-seeking and non-treatment-seeking populations. Addiction 2002; 97: 279-292

${ }^{39}$ McCambridge J, Strang J. The efficacy of single-session motivational interviewing in reducing drug consumption and perceptions of drugrelated risk and harm among young people: results from a multi-site cluster randomized trial. Addiction 2004; 99 (1): 39-52

${ }^{40}$ McCambridge J, Strang J. Deterioration over time in effect of Motivational Interviewing in reducing drug consumption and related risk among young people. Addiction 2005; 100 (4): 470 - 478 